EPJ Web of Conferences 64, 08008 (2014)

DOI: $10.1051 /$ epjconf/ 20146408008

(C) Owned by the authors, published by EDP Sciences, 2014

\title{
The Herbig Ae SB2 system HD 104237
}

\author{
C. R. Cowley ${ }^{1, a}$, F. Castelli ${ }^{2, b}$, and S. Hubrig ${ }^{3, c}$ \\ ${ }^{1}$ University of Michigan, Ann Arbor, MI, USA \\ ${ }^{2}$ Osservatorio Astronomico di Trieste, Italy \\ ${ }^{3}$ Leibnitz Institut für Astrophysik, Potsdam, Germany
}

\begin{abstract}
We present here the most recent abundance analysis of this Herbig Ae system based on high-resolution UVES and HARPS spectra and the results of our magnetic field measurements using high-resolution spectra obtained with HARPSpol.
\end{abstract}

\section{Introduction}

HD 104237 (DX Cha) is the optically brightest of the young Herbig Ae stars. The SB2 system was studied by Böhm, et al. (2004) who found a 19.86 day period. The binary, and several close companions described by Grady, et al. (2004) belong to the $\epsilon$ Cha association, estimated to be 3 to 5 Myr old. Fang et al. (2013) showed that the SED shows strong emissions at wavelengths beyond ca. $2 \mu \mathrm{m}$, indicating a hot, optically thick inner disk. The SED is more like that of a flattened disk model than to a flaring disk.

\section{The abundance analysis}

Atmospheric parameters for the primary star were derived from the wings of $\mathrm{H} \delta$, and the ionization equilibrium, Fe. We found $T_{\text {eff }}=8250 \mathrm{~K}$, slightly cooler than the $8550 \mathrm{~K}$ of Fumel and Böhm (2012) and $\log (g)=4.2$ for the primary star. The properties of the secondary star were also adjusted slightly from previous work. We adopted $T_{\text {eff }}=4800 \mathrm{~K}$, and $\log (g)=3.7$. Equivalent widths of the primary were corrected for the flux of the secondary from measurements of line strengths of both stars. An example is shown in Fig. 1. The synthesis calculations added spectra for the primary and secondary, and fit the composite spectrum.

Abundances were found for 25 elements in the primary and 17 in the secondary. Both equivalent width and spectral synthesis techniques were used. Abundances were solar, apart from lithium, and zirconium which was found to be 0.4 dex above solar. We found no indication of a systematic depletion of refractory elements as has been found in some Herbig Ae stars. Details are published elsewhere (Cowley, et al. 2013).

\footnotetext{
ae-mail: cowley@umichledu

be-mail: castelli@oats.inaf.it

ce-mail: shubrig@aip.de
}

This is an Open Access article distributed under the terms of the Creative Commons Attribution License 2.0, which permits unrestricted use, distribution, and reproduction in any medium, provided the original work is properly cited. 


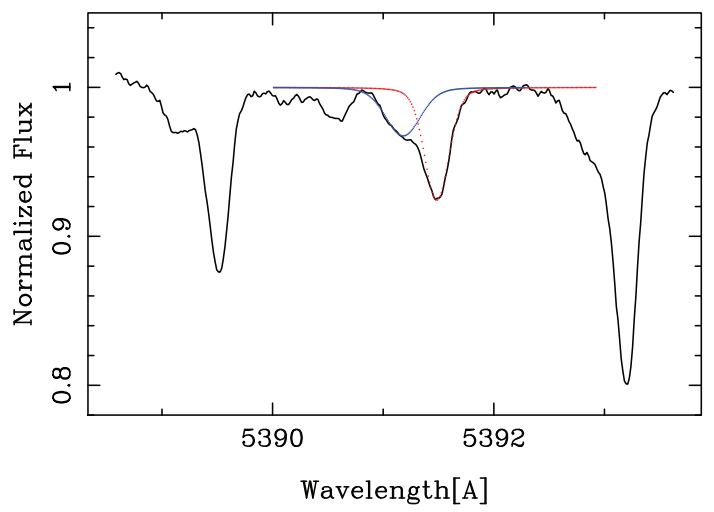

Figure 1. Measurement of Fe I $\lambda 5391.5$ in the primary (red/gray) and secondary (blue/light gray) spectrum.
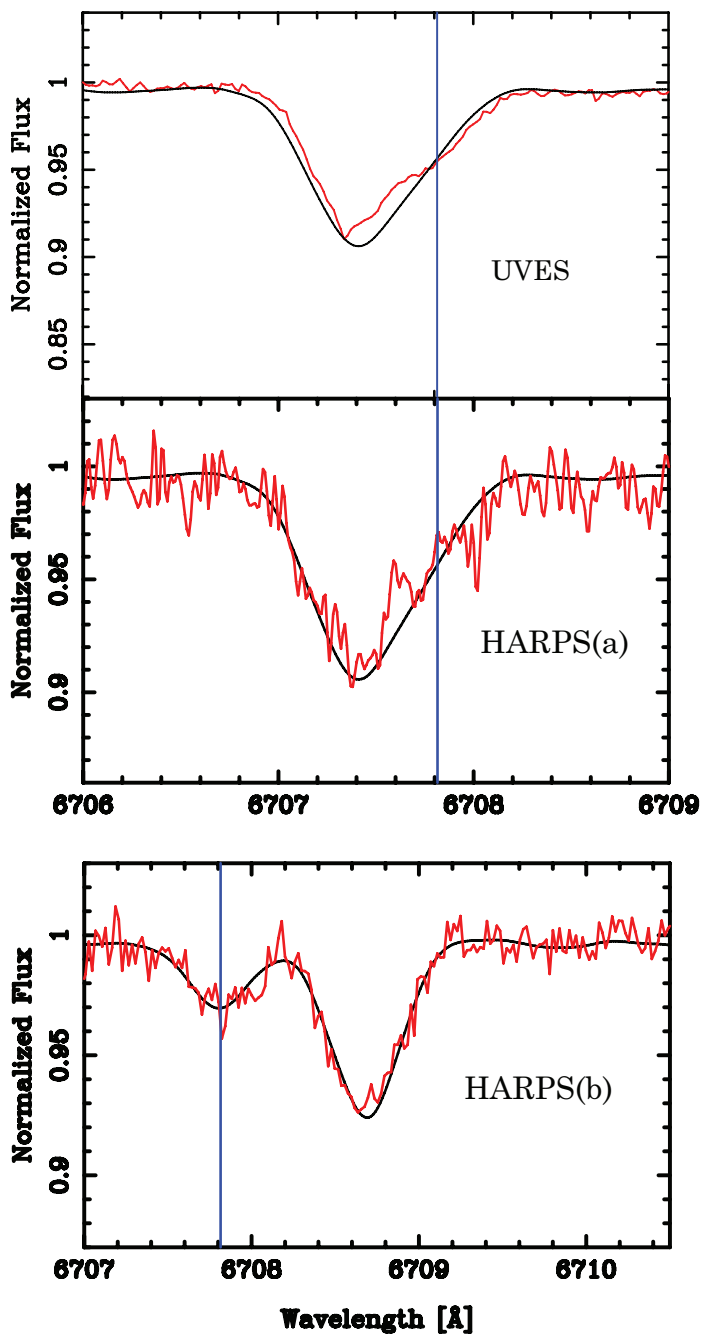

Figure 2. We show here the observed Li I region for different epochs: UVES observations in 2003, HARPS(a) observations in 2007, and HARPS(b) observations in 2010 , along with synthetic fits. The vertical lines mark the rest wavelength of $\mathrm{Li} \mathrm{I}$ in the primary. The stronger Li I absorption is due to the cooler secondary. Note that because of the orbital motion, the relative positions of primary and secondary absorption are reversed in the lower plot. Lithium appears to be somewhat enhanced, relative to the meteoritic value in the primary star. It is slightly depleted in the cooler secondary. 

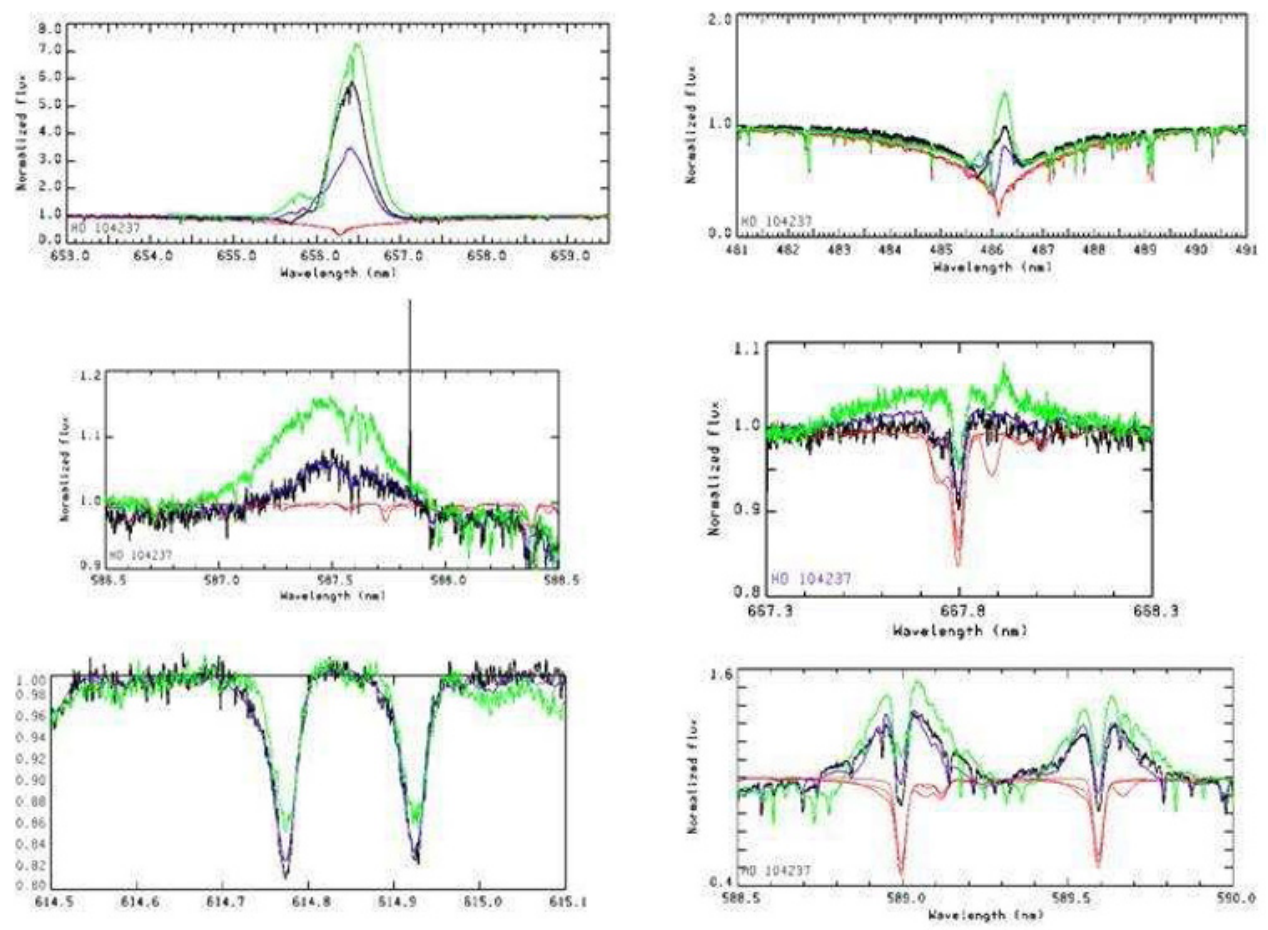

Figure 3. Illustrating variable lines. Upper: $\mathrm{H} \alpha$ and $\mathrm{H} \beta$; middle: $\mathrm{He} \lambda 5876$ [A] and 6678 [A]; bottom: Fe II-74, and $\mathrm{Na}$ I D. Note that the wavelength scale is in nanometers.

\section{Spectral variability}

The observations of HD 101412 at three different epochs (in 2003, 2007, and 2010) show that strong spectral lines exhibit significant variability. In Fig. 3 we show a few examples of variability. In a colorized version one may see profiles from 2003 (purple), 2007 (black), and 2010 (green). Red lines are for a spectrum computed in LTE.

\section{Magnetic field measurements}

Due to the very low projected rotational velocity $\left(v \cdot \sin (i)<9 \mathrm{~km} \mathrm{~s}^{-1}\right)$ of the primary, the accuracy of the magnetic field measurements in this star is rather high. Using high-resolution HARPSpol spectra, we were able to measure a field of $63 \pm 15 \mathrm{G}$, at a significance level of $4.2 \sigma$. In Fig. 4 we contrast the sharp, upper spectrum of HD 104237 with a more rapidly rotating magnetic Herbig Ae/Be star, HD 58647.

\section{Summary}

Presently, only a very few magnetic Herbig Ae/Be stars have both detailed abundances and magnetic field measurements. Further studies are important to better understand the disk-magnetosphere interactions of these stars. 

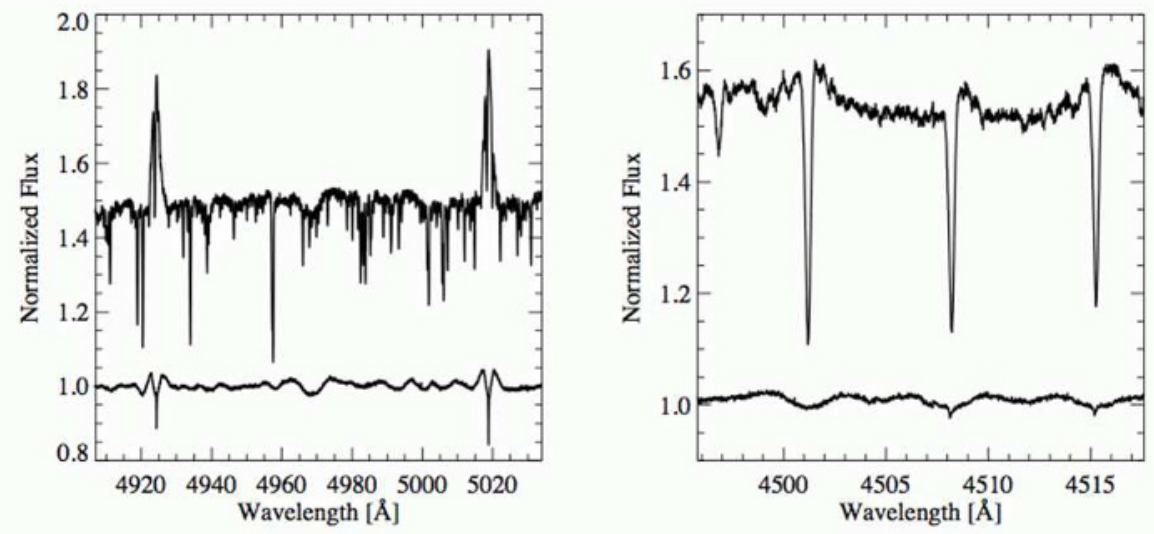

Figure 4. Illustrating the sharpness of the lines.

\section{References}

[1] Böhm, T., Catala, C., Balona, L. \& Carter, B. 2004, A\&A, 427, 907

[2] Cowley, C. R., Castelli, F. \& Hubrig, S. 2013, MNRAS, 431, 3485

[3] Grady, C. A., et al. 2004, ApJ, 608, 809

[4] Fang, M., van Boekel, R., King, R. R., et al. 2013, A\&A, 549, 15

[5] Fumel, A. \& Böhm, T. 2012, A\&A, 540, 108 